\title{
The focusing of weak shock waves
}

\author{
By B. STURTEVANT AND V. A. KULKARNY \\ Graduate Aeronautical Laboratories, California Institute of Technology, Pasadena
}

(Received 23 May 1975)

This paper reports an experimental investigation, using shadowgraphs and pressure measurements, of the detailed behaviour of converging weak shock waves near three different kinds of focus. Shocks are brought to a focus by reflecting initially plane fronts from concave end walls in a large shock tube. The reflectors are shaped to generate perfect foci, arêtes and caustics. It is found that, near the focus of a shock discontinuity, a complex wave field develops, which always has the same basic character, and which is always essentially nonlinear. A diffracted wave field forms behind the non-uniform converging shock; its compressive portions steepen to form diffraction shocks, while diffracted expansion waves overtake and weaken the diffraction shocks. The diffraction shocks participate in a Mach reflexion process near the focus, whose development is determined by competition between the convergence of the sides of the focusing front and acceleration of its central portion. In fact, depending on the aperture of the convergence and the strength of the initial wave, the three-shock intersections of the Mach reflexions either cross on a surface of symmetry or remain uncrossed. In the former case, which is observed if the shock wave is relatively weak, the wavefronts emerge from focus crossed and folded, in accordance with the predictions of geometrical acoustics theory. In the latter, the strong-shock case, the fronts beyond focus are uncrossed, as predicted by the theory of shock dynamics. It is emphasized that in both cases the behaviour at the focus is nonlinear. The overtaking of the diffraction shocks by the diffracted expansions limits the amplitude of the converging wave near focus, and is the mechanism by which the maximum amplification factor observed at focus is determined. In all cases, maximum pressures are limited to rather low values.

\section{Introduction}

In unsteady flows with shock discontinuities, it very often happens that the shock wavefronts become curved. For example, plane shockfronts are deformed by propagation through inhomogeneous or moving media, or by reflexion from curved surfaces. Current applications in gasdynamics, in which such conditions prevail, range from pulsed gas lasers to the sonic boom. 'This paper is concerned with the behaviour of weak shocks curved concave toward the direction of propagation. Such waves, once formed, converge and tend to focus. For discontinuous fronts, the only counterbalancing mechanism is smoothing by nonlinear wave propagation transverse to the incident front, responsible for the stability of plane shocks (Whitham 1957). This effect is weak for weak waves, so the amplitude of 

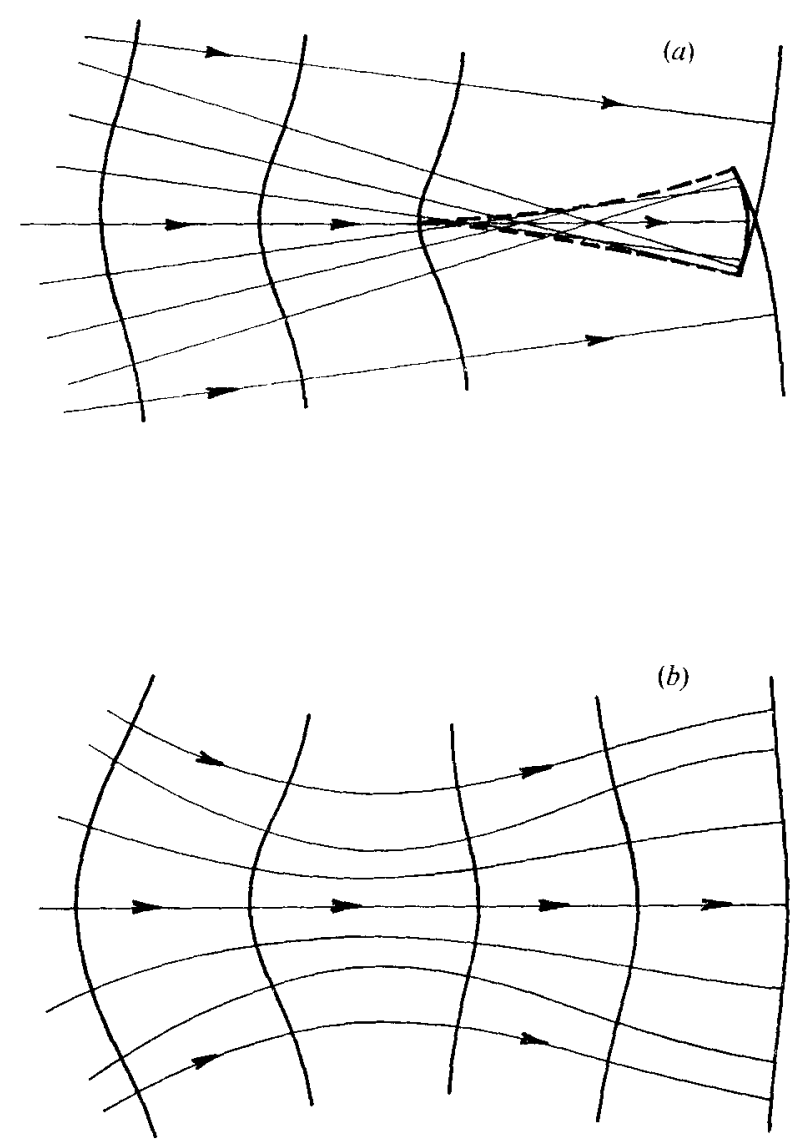

Figure 1. Focusing of a weak shock: (a) according to geometrical acoustics (linear); (b) according to shock dynamics (nonlinear).

such shocks can become greatly magnified. It often becomes both important and necessary in such cases to predict the maximum amplitude and the rather significant changes of wavefront geometry that may occur in the focal region. As will be seen below, the predictions of purely linear theory (geometrical acoustics) are qualitatively quite different from the predictions of theories which include the effects of gasdynamic nonlinearity.

This paper reports an experimental investigation, using shadowgraphs and pressure measurements, of the detailed behaviour of converging weak shocks near three different kinds of focus: caustics, arêtes and perfect foci. Whereas previous investigations have shown that far beyond the focus the wavefront geometry and amplitude may agree with the predictions of geometrical acoustics when the waves are initially weak, or with nonlinear theory when the waves are strong, the central result of the present investigation is that near the focus the same complex wave field always develops, owing to nonlinear effects. Increased sound speed and nonlinear steepening in the non-uniform field behind the converging incident front affect the development of the incident wave, and of a set of diffracted 
waves in the focal region. These effects determine the maximum amplitude at focus, and also even the asymptotic behaviour far beyond the focus.

\subsection{Geometrical acoustics}

The description of the behaviour of an acoustic discontinuity (acoustic shock) is provided by the geometrical acoustics of Keller (1954). The focusing of such a wave is shown in figure $1(a)$, in which the shock is shown moving to the right, at successive instants. The front propagates normal to itself with a fixed speed at all times. In an isotropic atmosphere the trajectories of points on the shock, or rays, are straight lines normal to the shockfront, and, for a concave shockfront, cross, as shown in the figure. The surface on which adjacent rays cross is called a caustic surface. In many cases, the shockfront has a minimum radius of curvature, which leads to a cusp in the caustic, called an arête, also shown. According to geometrical acoustics, the wave amplitude is inversely proportional to the square root of the ray-tube area. So, when the rays cross, infinite amplitude is predicted. Evidently geometrical acoustics is not valid near such singular regions because it assumes small amplitudes to begin with. None the less, it provides the simplest description of the geometry of the initial wavefront and, to some extent, of the process of focusing.

As the wrinkled wavefront propagates, its concave portions strengthen while the convex portions weaken, so the shock strength becomes non-uniform along the front. The non-uniform pressures in the fluid behind the wavefront due to the variation of shock strength along the front are supported by a diffracted wave field. In general, expansion waves travel into the high-pressure fluid behind concave fronts and compression waves into fluid behind convex fronts. These waves are responsible for the gradual equilibration of pressure far downstream of the fronts. It turns out that they also play an important role at a focus.

Three kinds of foci may be identified: caustics, arêtes, and perfect foci. Let the radius of curvature $R(s, t)$ of the travelling shockfront be expressed as a function of arc length $s$ along the initial shock and of time $t$, i.e.

$$
R(s, t)=R(s, 0)-c t .
$$

( $c$ is the speed of the shockfront.) Then, (i) $R(s, t)=0$ implies a caustic; (ii) $R(s, t)=\partial R(s, t) / \partial s=0$ implies an arête; and (iii) $R(s, t)=0$ for all $s$ in some non-zero range of $s$ implies a perfect focus. If there are no singularities on the initial shockfront $(R(s, 0) \neq 0$ for all $s)$, then it is clear that a local minimum of $R$ leads to an arête. In most applications, one would expect $R$ to be a smooth, continuous function of $s$ with a minimum. For example, a large, plane shockfront will be locally dimpled when it interacts with a small inhomogeneity. Thus, one might expect the arête to be the most-frequently observed focus. A starting point of a caustic or a perfect focus are rather special circumstances. Indeed, for a caustic to have an endpoint, $R$ must be discontinuous on the ray passing through the endpoint. The wavefront folds on this ray, past the caustic, without becoming singular.

A perfect focus, an arête and the beginning of a caustic are inherently unsteady processes. However, in the latter two cases, if locally, near the caustics, the 
curvatures of the caustics and the rays remain constant, the processes become locally quasi-steady far from the first point of focus.

Acoustic theory predicts that, when a shock discontinuity passes through a perfect line focus, not only does its amplitude become infinite at the focus, but the shock changes into a logarithmically singular compression-expansion front beyond focus (Friedlander 1958). Furthermore, if the curvature in the other direction focuses this front again, it comes out inverted (i.e. an expansion shock).

\subsection{Nonlinear effects}

The major influence of finite amplitude is the increase of wave speed with amplitude; it is taken into account by Whitham's approach to shock dynamics (Whitham 1957, 1959). In this theory, a description similar to geometrical acoustics is used, with shockfronts and with rays orthogonal to the fronts. However, the shockfront can travel at different speeds along different rays depending on its amplitude. This effect turns the shockfront and bends the rays. The concave portion of the shock amplifies and accelerates relative to the not-so-concave portions. The rays then curve away from the focus and the shockfront becomes 'smooth', as illustrated in figure $1(b)$. The rays do not cross, and the amplitudes are finite. The shock emerges flattened, and it has no loop.

The possibility that two different wavefront geometries might be observed beyond the focus recalls another problem in which the same behaviour is observed: namely, diffraction of a shock wave over a wedge (Bleakney \& Taub 1949). Depending on the shock strength and wedge angle, either regular reflexion or Mach reflexion is observed. Indeed, acoustic regular reflexion can be treated by acoustic theory (Keller \& Blank 1951) and nonlinear Mach reflexion, by shock dynamics theory (Whitham 1957). In light of this similarity and of what is known about shock diffraction, it is reasonable to expect that the formation of threeshock intersections will also be important to the behaviour of shocks near foci. The present results show that the formation of looped and crossed wavefronts corresponds to regular reflexion from surfaces of symmetry, while uncrossed wavefronts correspond to Mach reflexion.

\subsection{Previous investigations}

Friedman, Kane \& Sigalla (1963) have extended the theory of shock dynamics in order to apply it to focusing by atmospheric refraction. It has been pointed out by M. P. Friedman \& Chou (1965), and independently by M. B. Friedman (1968), that shock-dynamic computations fail to show focusing near the caustic. For steady caustics, Guiraud (1965) and Hayes (1968) hypothesized a nonlinear similarity behaviour near the singular region, based on the transonic flow equations. Using these results, Seebass, Murman \& Krupp (1970) and Gill (1973) obtained solutions for the nonlinear behaviour of shocks near caustics.

Experimental evidence of loops and folds in wavefronts beyond focus has been obtained in flight tests (e.g. Wanner et al. 1972) and in the laboratory (Beasley, Brooks \& Barger 1969; Thery, Peter \& Pfister 1970; Cornet 1972). On the other hand, behaviour that seems to be dominated by nonlinear effects has also been observed. In an investigation of shock stability, Briscoe \& Kovitz (1968) photo- 
graphed perturbed shocks. These pictures show shockfronts coming out of foci without any folds. Naumann \& Hermanns (1974) have observed shockfronts near caustics during their investigation of the interaction between a shock wave and a vortex field. Here also, the shockfronts have no loops or folds, but they have three-shock intersections. Sanai \& Toong (1974) produced foci by refraction inside a ballistics range, and found no evidence of folding near arêtes.

Thus, previous experimental and theoretical evidence has indicated that one of two quite different wavefront configurations might be observed far beyond focus, depending on the initial conditions. The fact that waves conforming to the predictions of linear theory (i.e. with looped and folded fronts) are observed for weak shocks has been taken to mean that in these circumstances the linearizing approximation is sufficient to describe the entire focusing process. A disturbing consequence of this conclusion is that, in order to avoid the singularity at the focus, the assumption of a shock discontinuity must be discarded; the shock amplitude evidently is limited by linear diffraction effects at distances of the order of the shock thickness from the focus, i.e. where geometrical acoustics ceases to be valid and where wave acoustics holds (Obermeier 1974). Since in most applications the shock waves are very thin compared with a length scale characteristic of the focusing geometry (e.g. the initial radius of curvature of the concavity in the shock), this conclusion would suggest that very large amplifications occur (even though such amplifications have not actually been measured). In the present investigation, the behaviour at focus was observed in much finer detail than previously. The central result, as mentioned above, has been the demonstration: for thin shocks nonlinearity limits wave amplitudes before linear diffraction effects do, even when looped and folded fronts are observed beyond focus. The mechanism by which this is accomplished is exhibited.

\section{Experimental}

The GALCIT $17 \mathrm{in}$. dia. shock tube, originally designed for studies of rarefied gasdynamics (Liepmann et al. 1962), was used, owing to its conveniently large size, and the excellent repeatability of the strength and planarity of the shock waves it produces. In these experiments, weak shocks in atmospheric air were generated by compressed nitrogen as the driver gas. The shock Mach numbers varied from 1.005 to $1 \cdot 5$, as shown in table 1 .

The incident shock waves were brought to a focus by reflecting them from concave reflectors at the end wall of the shock tube (figure 2). The focal regions are viewed through $8.4 \mathrm{~cm}$ dia. windows, and the reflectors are positioned along the shock-tube axis relative to the windows so that the region of interest is visible. Cylindrical reflectors were constructed of machined wood blocks, smoothed and polished with sanding sealer. Parabolic reflectors, producing perfect line foci, were machined following templates, whereas more complex shapes producing caustic surfaces and their cusp lines were formed in accordance with specified co-ordinates. A paraboloidal reflector producing a point focus was also constructed by embedding a commercial silvered glass mirror in epoxy on a wooden backing. Table 2 lists the various reflectors and their important characteristics. 


\begin{tabular}{|c|c|c|c|}
\hline \multirow[b]{2}{*}{$M_{a}$} & \multicolumn{2}{|c|}{ Diaphragm } & \multirow[b]{2}{*}{$P_{1}$ (atm) } \\
\hline & Material & Thickness $(\mathrm{cm})$ & \\
\hline $1 \cdot 005$ & & 0.0006 & $1 \cdot 0$ \\
\hline $1 \cdot 01$ & Mylar & 0.0013 & 1.0 \\
\hline 1.02 & & 0.0025 & $1 \cdot 0$ \\
\hline 1.03 & & 0.0076 & $1 \cdot 0$ \\
\hline $1 \cdot 1$ & & 0.015 & 1.0 \\
\hline 1.2 & Al 1100-0 & 0.025 & $0 \cdot 67$ \\
\hline $1 \cdot 3$ & & 0.051 & $0 \cdot 67$ \\
\hline 1.5 & & 0.051 & 0.33 \\
\hline
\end{tabular}

TABLE 1. Conditions for producing various shocks in the GALCIT 17 in. shock tube.

\begin{tabular}{|c|c|c|c|c|c|}
\hline No. & Shape & $\begin{array}{l}\text { Width } \\
\text { (cm) }\end{array}$ & Focus & $\begin{array}{l}R_{\min } \\
(\mathrm{cm})\end{array}$ & $\begin{array}{c}\text { Angle of } \\
\text { convergence } \\
\text { (deg) }\end{array}$ \\
\hline 1 & Parabolic cylinder & $20 \cdot 32$ & Perfect line focus & 6.03 & 160 \\
\hline 2 & Parabolic cylinder & $20 \cdot 32$ & Perfect line focus & $12 \cdot 06$ & 91 \\
\hline 3 & Parabolic cylinder & $20 \cdot 32$ & Perfect line focus & $24 \cdot 13$ & 48 \\
\hline 4 & $\begin{array}{l}\text { Axisymmetric } \\
\text { paraboloid }\end{array}$ & $20 \cdot 32$ & $\begin{array}{l}\text { Perfect point } \\
\text { focus }\end{array}$ & $6 \cdot 03$ & 160 \\
\hline 5 & $\begin{array}{l}\text { Asymmetric } \\
\text { concave }\end{array}$ & $23 \cdot 5$ & $\begin{array}{l}\text { Caustic with } \\
\qquad R=8.26 \mathrm{~cm}\end{array}$ & $6 \cdot 76$ & 100 \\
\hline 6 & $\begin{array}{l}\text { Concave with } \\
\text { flat ends }\end{array}$ & $21 \cdot 6$ & Arête & $2 \cdot 54$ & 120 \\
\hline
\end{tabular}

TABtis 2. Reflectors and their characteristics. All reflectors had sharp edges, except no. 6, which gradually became convex and filled the whole cross-section of the tube. It was designed to produce a concave acoustic reflexion of a shape $x=(1 \cdot 27) y^{2}-\left(7 \cdot 41 \times 10^{-4}\right) y^{4}$ (in cm).

$A$

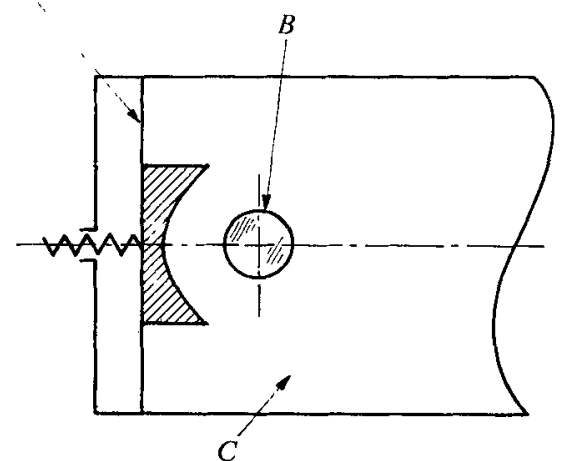

A

(b)

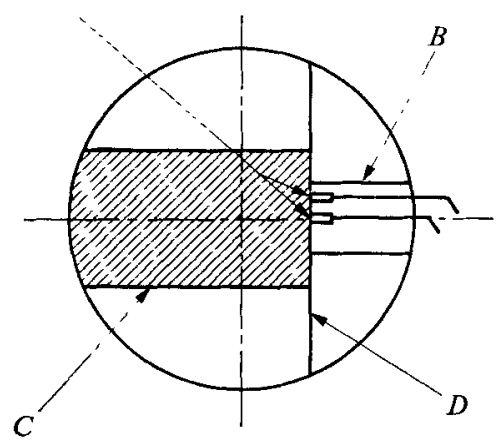

Figure 2. Schematic diagram of apparatus. (a) For shadowgraphs. $A$, moveable endwall with reflector. $B, 8.4 \mathrm{~cm}$ dia. window. $C, 17 \mathrm{in}$. dia. shock tube. (b) For pressure measurement: view from diaphragm. $A$, Pressure transducers. $B, 8.4 \mathrm{~cm}$ dia. instrument capsule. $C$, Reflector. $D$, Dividing plane. 
The cross-sections of the reflectors, viewed in the plane parallel to the viewing window, do not span the entire diameter of the shock tube, but have a height of only $20 \mathrm{~cm}$. They all have sharp corners (as indicated schematically in figure 2), except for reflector no. 6, as described in table 2. To examine the effect of the sharpness of the corners of the reflectors, experiments were conducted with reflector no. 1 , in which the gap between reflector and shock tube wall was completely filled in with either a flat baffle or a rounded baffle eliminating any discontinuity of slope in the reflector surface (Kulkarny 1975). These changes had very little effect on the shape of the wavefronts and other features near focus.

The reflected shock waves were observed with spark shadowgraphs. The spark source has a duration of less than a microsecond. A mirror of $1.4 \mathrm{~m}$ focal length collimates the beam from the source. The aperture at the source is $\frac{1}{4} \mathrm{~mm}$ in diameter. The viewing windows are made of flat optical glass and are mounted so that perturbation of the smoothness of the shock-tube walls is minimized. A single shadowgraph is obtained for each run of the shock tube. By changing a time delay between the occurrence of a trigger signal from a fixed piezoelectric transducer which detects incident shock arrival and the initiation of the spark, it is possible to make repeated runs to map out the entire focusing process.

Pressure histories are measured at various points in the flow field using small piezoelectric transducers $(2.5 \mathrm{~mm}$ dia., $1 \mu$ s rise time) mounted flush with the surface of a dividing plane aligned parallel with the viewing windows (figure 2).

\section{Results}

\subsection{Perfect focus}

3.1.1. Shadowgraphs. Figure 3 (plate 1) shows the reflexion from a parabolic cylinder (reflector no. 1, table 2) of an incident plane shock of $M_{s}=1.1$ at successive instants of time. In this sequence the reflexion travels from left to right, and two different window locations are used. In figures $3(a)$ and $(b)$, the reflector is at the immediate left of the frame. In figures $3(c)-(f)$ it has been moved to the left $4.5 \mathrm{~cm}$, out of the field of view.

In figure $3(a)$ the wave pattern has three distinguishable components. The dark circular wavefront in the centre, concave to the right, is the reflected shock travelling towards the focus. Extending from this wavefront on either side, are dark convex (to the right) compressive diffracted fronts emanating from the sharp corners of the parabolic reflector. Finally, behind these waves (on the left), are two circular light wavefronts, travelling towards the axis of symmetry. These are expansion waves, the remainder of the disturbance generated by the corners of the reflector.

In figure $3(b)$ the shockfront has converged towards the focus, and the diffracted expansions behind it have just passed through each other; the expansion from the lower corner of the reflector (convex upwards, travelling up) in the upper half of the picture is the same wave as that in the lower half of figure $3(a)$. This picture exhibits an important nonlinear effect. The intersections of the expansion waves with the shockfront have met each other before the shockfront has reached the focus. 
In figure $3(c)$ the shock is at the focus. In the rest of the sequence it is seen coming out of the focus with a crossed and folded configuration, typical of the behaviour of weak shocks, leaving behind a region of hot gas at the focus. The compressive diffracted fronts from the corners of the reflector have crossed in front, and thus precede the focused shock, which follows between the folds. The two tails of the dark focal hot spot are the slipstreams of the folds in the shock which, as can be seen, have formed into three-shock intersections. This has occurred because of the appearance of shocks outside the folds, the most important feature of the entire focusing process. These shocks can be seen forming in figure $3(b)$ ahead of the diffracted expansion fronts, and can be followed throughout the rest of the sequence. Henceforth they will be referred to as diffraction shocks. They form by nonlinear steepening of a compressive wave field in the fluid behind the incident front which, though initially not visible in the shadowgraphs, is exhibited by the pressure measurements presented below. The slipstreams at the intersection of the diffraction shock, the shock emerging from the focus and the compressive diffracted front from the corner form the boundaries of the focal region. They can be identified with the shock-shocks of the theory of shock dynamics. The shock-shocks initially cross, and when they turn around and cross again, they enclose the focal spot. From this point on, crossed and folded shockfronts are seen.

Figure 4 (plate 2) shows the reflexion of a plane shock of strength $M_{s}=1 \cdot 3$, in the same experimental configuration. This sequence of shadowgraphs shows strong-shock $\dagger$ behaviour. Again, the intersections of the diffracted expansion waves with the shock meet and turn into three-shock intersections before the shockfront gets to the focus, but it occurs much earlier in this case. The stem shock of the intersections is surprisingly plane, and leaves the fluid in the focal spot with a higher entropy than its surroundings. In contrast to the weak-shock case, the shock-shocks do not turn around and cross a second time but simply spread apart, so the focal spot never closes. Consequently, the configuration of waves beyond focus is not crossed and folded, but is flattened. In figures $4(f)$ and $(g)$ the waves have left the field of view, and only the slipstreams of the threeshock intersections are seen. The slipstreams evidently become unstable. In fact, at the beginning of the focal spot the layers cross, and in figure $4(g)$ this crossing appears to be forming into a jet.

A selection of five such shadowgraph sequences for five different incident shock strengths is shown in figure 5 (plate 3 ). The sequences of figures 3 and 4 are third and fifth from the top, respectively. The fourth sequence, $M_{s}=1 \cdot 2$, shows a borderline case between crossing and not crossing of the shockfront. In this case, the shock-shocks turn around and come very close to a second crossing, but then move away. The focal spot is almost pinched off at that point, but not quite, so the waves leaving the focus are not crossed. For shocks weaker than this the shockshocks cross twice, giving a finite focal spot and crossed shockfronts. The stronger

$\dagger$ For simplicity, we use terms of relative strength to denote the different regimes of shock behaviour, even though 'strong' may at times refer to, e.g., a $M_{s}=1 \cdot 1$ shock wave, and though, as will be seen in \$3.1.3, the behaviour depends on the aperture of the con. vergence in addition to shock strength. 


\begin{tabular}{|c|c|c|c|c|c|c|}
\hline \multirow[b]{2}{*}{ No. } & \multirow[b]{2}{*}{ Type of focus } & \multicolumn{3}{|c|}{ Observations } & \multicolumn{2}{|c|}{ Computations } \\
\hline & & $\underset{\text { (in.) }}{f_{\min }}$ & $\begin{array}{c}\text { Angle of } \\
\text { convergence } \\
\text { (deg) }\end{array}$ & $\begin{array}{c}M_{t} \\
\text { (value or } \\
\text { range) }\end{array}$ & $\begin{array}{c}\lambda I_{t} \\
\text { (value or } \\
\text { range) }\end{array}$ & $\begin{array}{c}\text { Angle of } \\
\text { convergence } \\
\text { (deg) }\end{array}$ \\
\hline 1 & Perfect line focus & $2 \cdot 375$ & 160 & $1 \cdot 2$ & $1 \cdot 22$ & $160 \cdot 3$ \\
\hline 2 & Perfect line focus & $4 \cdot 75$ & 91 & $1 \cdot 03-1 \cdot 1$ & $1 \cdot 05-1 \cdot 075$ & $91 \cdot 5$ \\
\hline 3 & Perfect line focus & $9 \cdot 5$ & 48 & $1 \cdot 01-1 \cdot 02$ & $1 \cdot 01-1 \cdot 02$ & $45 \cdot 7$ \\
\hline 4 & Perfect point focus & $2 \cdot 375$ & 160 & $1 \cdot 03-1 \cdot 1$ & & \\
\hline 5 & Smooth caustic & $2 \cdot 66$ & 100 & Not measured & & \\
\hline 6 & Cusped caustic (arête) & $1 \cdot 00$ & 120 & $1 \cdot 3-1 \cdot 5$ & & \\
\hline
\end{tabular}
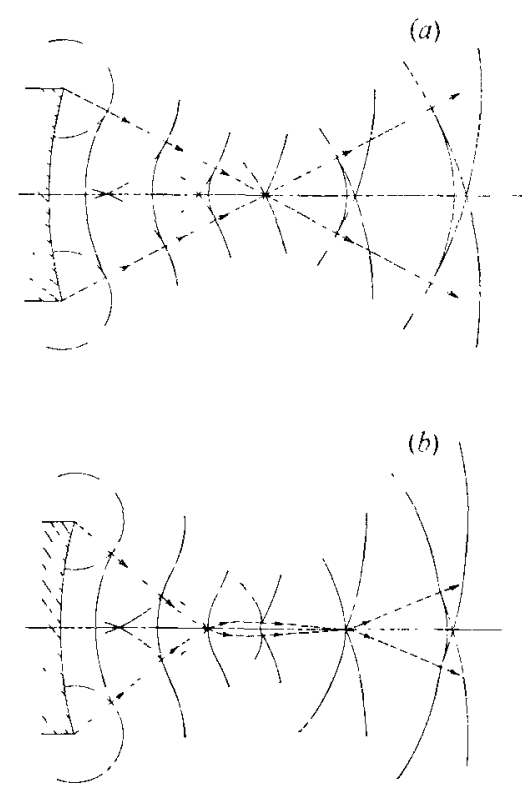

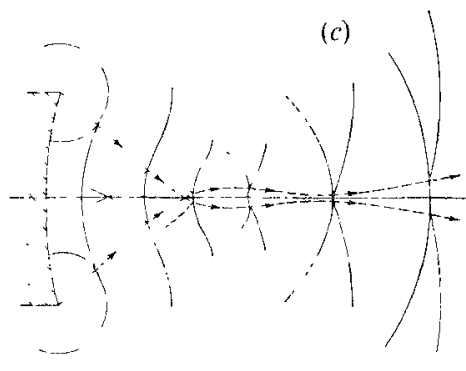

(d)

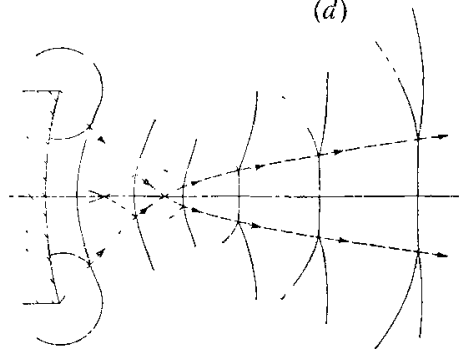

Figure 6. Schematic representation of the effect of the shock strength on focusing. Perfect focus of: $(a)$ sound pulses; $(b)$ weak shocks; $(c)$ moderately strong shocks; $(d)$ strong shocks.

the shock, the larger the focal spot and the smaller the triangular loop in the shockfront. For shocks stronger than the transition shock strength (in this case, $M_{s}=1 \cdot 2$ ), the focal spot becomes semi-finite and the loop disappears. In the focal spot, the shock is plane and normal, and the amplitude is finite. Thus, nonlinearity spreads the focus and gives a finite maximum amplitude. The measured conditions at which transition from crossed to uncrossed wavefronts occurs in our experiments are tabulated in table 3.

Figure 6 summarizes the results schematically, showing a perfect focus of $(a)$ sound pulses, $(b)$ weak shocks, $(c)$ moderately strong shocks and $(d)$ strong shocks. The typical wavefront patterns for each are shown by solid lines. The 


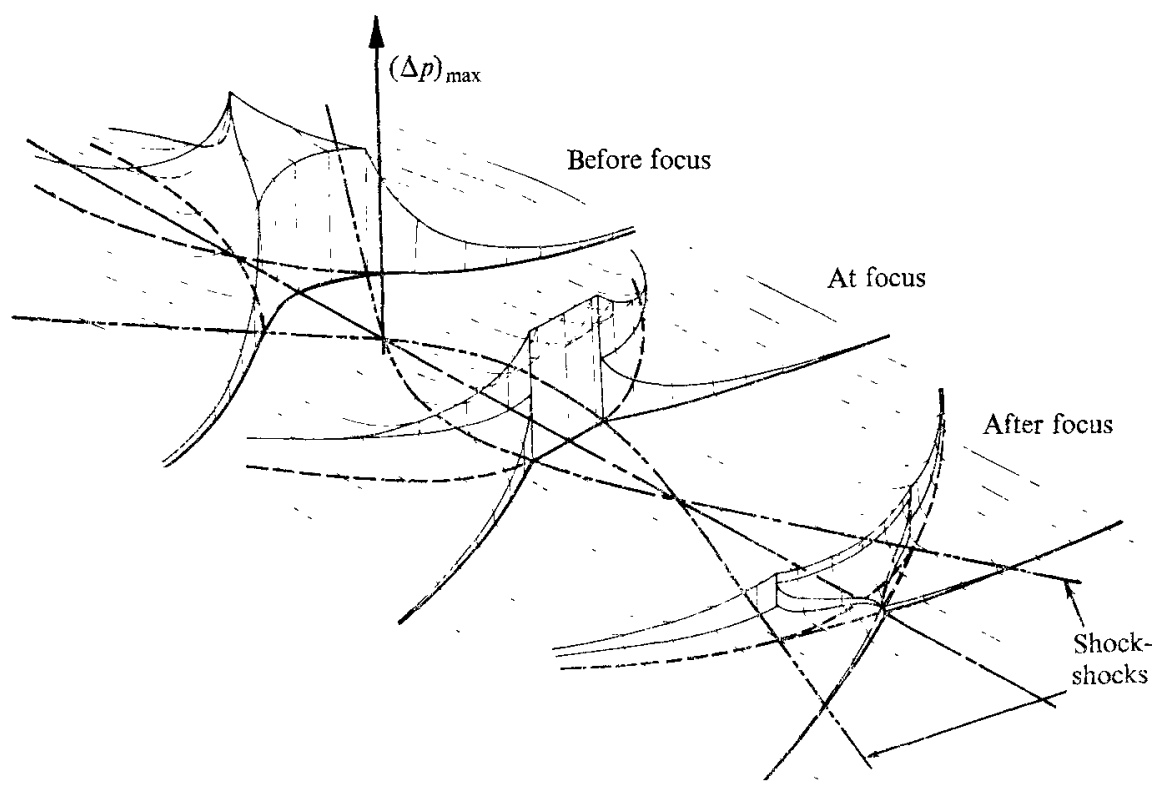

FiguRE 8. Three stages of the pressure field near a perfect line focus. Qualitative isometrio views are shown for waves travelling towards and to the right of the observer. Wavefronts are shown as heavy lines on the ground, and pressure is vertically upwards.

trajectories of the three-wave intersections are shown by dashed lines for each case. In the limit of infinitesimal amplitude, these curves reduce to the rays of geometrical acoustics that confine the focusing shock.

3.1.2. Pressure histories. The trajectories of the three-wave intersections divide the flow field into regions that can be identified with the kinds of waves occurring in them. Each of the regions exhibits a characteristic pressure history. These pressure histories have been measured and are shown in figure 7 (plate 4). On the pressure traces, time increases to the right (abscissa), and pressure increases upwards (ordinate). Shocks are seen as discontinuities of pressure, whereas diffracted waves appear as discontinuities of slope. The reader may identify the different pressure variations with the corresponding wavefronts shown on the figure. (For points closest to the reflector, the first pressure jump on the traces corresponds to the incident shock.) Even though only the weak-shock case is shown, the pressure traces remain qualitatively the same in all cases, though for strong shocks the focal region extends to infinity, so crossed shockfronts and the corresponding pressure traces do not appear.

A great deal of pressure variation can be seen between the wavefronts. The pressure rises smoothly behind the leading shockfront in all regions except the focal region; as described above, the compressions behind the fronts steepen to form the diffraction shocks. After the last wavefront the pressure invariably drops in all regions. Of even more significance is the sharp expansion following the strong stem shock in the focal region.

It is useful to visualize the instantaneous pressure field throughout the focal region by cross plotting the pressure histories of figure 7 in a three-dimensional 


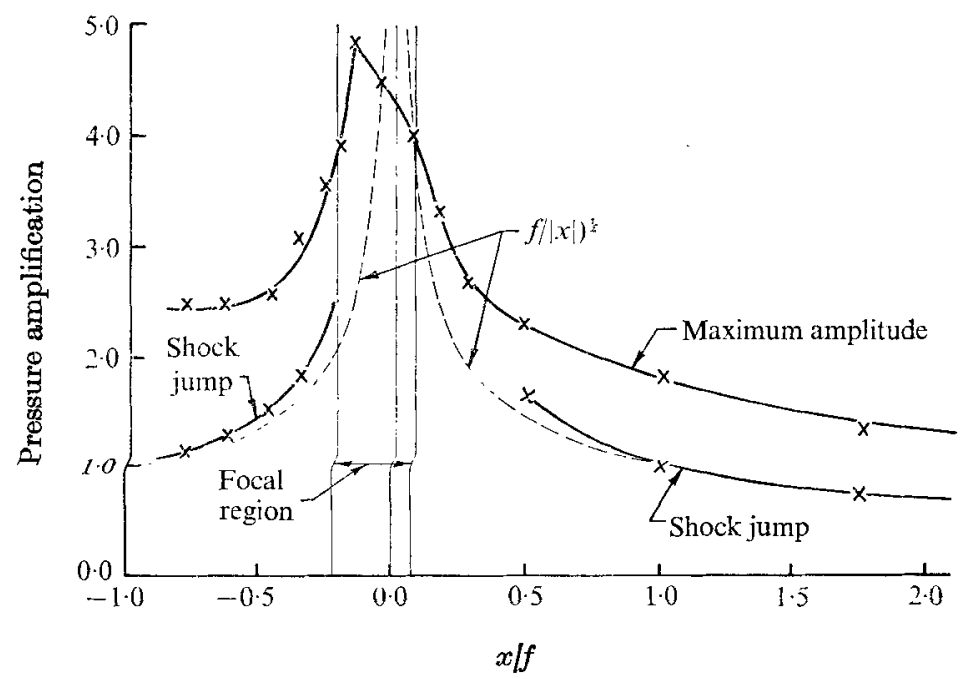

FIgURe 9. Maximum pressure and shock jump at points along the axis of symmetry for a perfect line focus; incident shock strength $M_{s}=1.1$ and reflector no. 1 . Distance from the focus is normalized with focal length $f$, and the pressure is normalized with the pressure jump of the converging shock as it leaves the reflector surface.

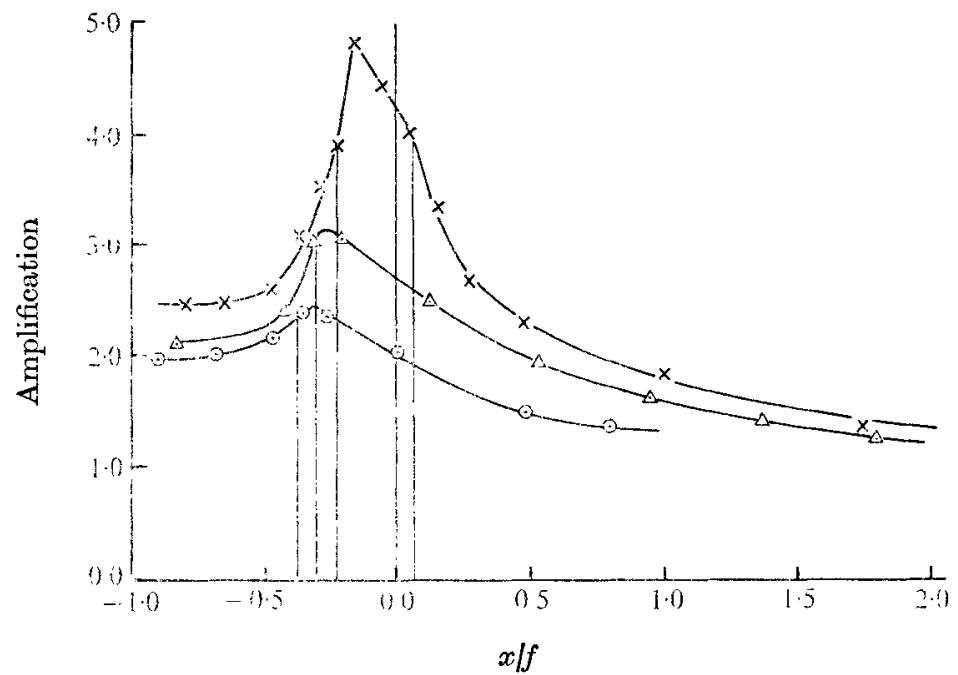

Frgure 10. Effect of shock strength on maximum pressure at points along the axis of symmetry of a perfect line focus. Scales defined as in figure $9 . M_{s}: \times, 1 \cdot 1 ; \Delta, 1 \cdot 2 ; 0,1 \cdot 3$.

view. A qualitative isometric view of three different wave patterns is shown in figure 8. The wavefronts (heavy lines) are seen on the ground, travelling towards and to the right of the observer. The pressure is shown vertically upwards. The light lines approximately represent the pressure histories observed at different points in the flow field. These views show that the sharp expansion following the stem shock in the focal region arises from a combination of the two expansion waves travelling behind the shock. (These waves are the diffracted expansion 


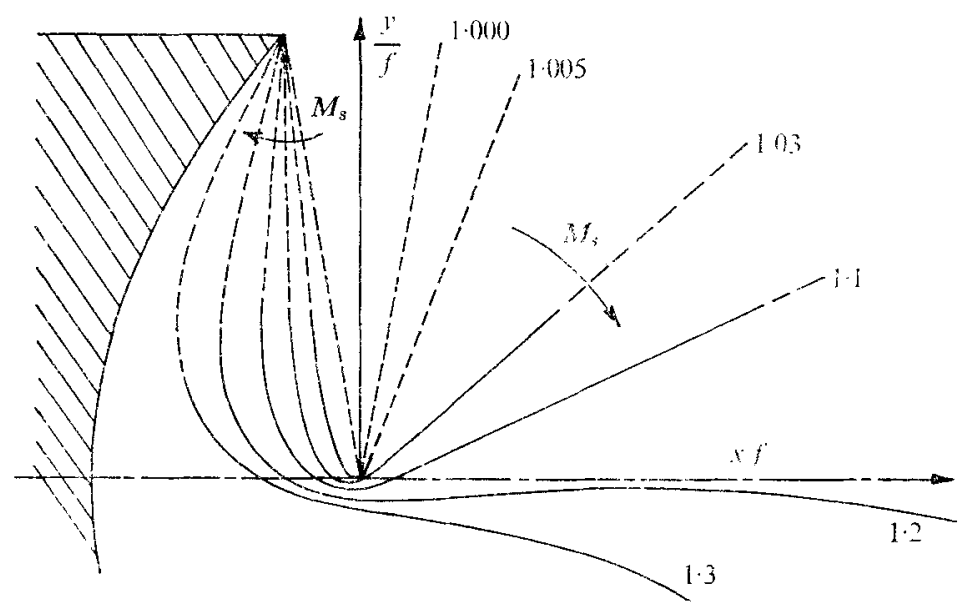

FIGURE 11. Influence of shock strength on the trajectories of three-shock intersections for a perfect focus (reflector no. 1).---, Unobserved parts.

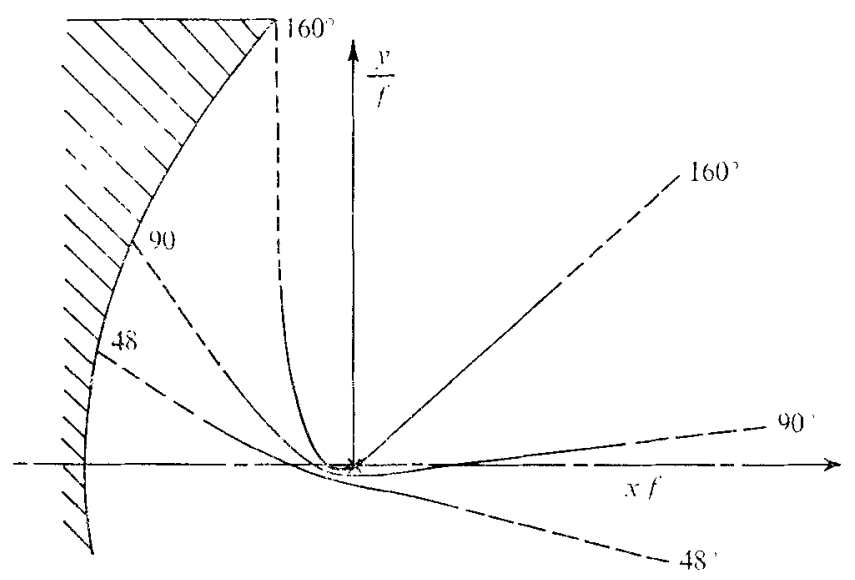

FIGURE 12. Influence of the aperture of convergence on the trajectories of three-shock intersections for a perfect focus. Incident shock strength $M_{s}=1 \cdot 03$. Both co-ordinates normalized with focal length. Apertures of 160,90 and $48^{\circ}$ correspond respectively to reflectors nos. 1, 2 and 3 .

fronts from the corners of the reflector.) Therefore, it is clear that the first crossing of the trajectories of three-wave intersections is the point of maximum amplitude; the amplitude is severely attenuated at later instants by the two overtaking diffracted expansions. The smooth compression due to non-uniform strength along the shockfront is also seen in the first view. In the second view it has steepened to form the diffraction shocks. Later, when the waves cross, the front coming out of the focus consists of a smooth compression, the top of which has formed into a shock, followed by an expansion. It is interesting to note that this wave corresponds to the logarithmic singularity of the acoustic approximation.

3.1.3. Maximum pressures and shock-shock trajectories. The maximum observed pressure and the amplitude of the shock jump (normalized with the amplitude 
of the converging shock as it leaves the reflector surface) on the axis of symmetry are plotted in figure 9 . The distance from the focus, along the axis, is normalized with the focal length, $f=6.0 \mathrm{~cm}$. As can be seen from figure 8 , outside the focal region the shock amplitude is less than the maximum pressure. It is surprisingly close to the $(f /|x|)^{\frac{1}{2}}$ dependence predicted by geometrical acoustics.

The dependence of maximum pressure on incident shock strength is shown in figure 10. For the stronger shocks, the diffracted expansion fronts from the reflector corners overtake the shock on the centre-line earlier and reduce its amplitude.

As has already been seen, the trajectories of the three-wave intersections are the most graphic manifestation of geometrical effects at the focus. Figure 11 shows the trajectories of three-wave intersections originating at one corner of the reflector for different incident shock strengths. The trajectories are traced directly from the shadowgraphs; the unobserved parts are shown dashed. For each trajectory, the region between the crossings with the axis of symmetry near the focus represents the focal region. In the acoustic limit the three-wave intersection occurs at the point of tangency between the shockfront and the diffracted expansion and its trajectory is the ray from the corner to the focus (shown dashed).

The results of experiments using shallower reflectors than reflector no. 1 are given in figure 12. It can be seen that for a smaller angle of convergence the focal region is larger, and that as the aperture decreases nonlinear effects increase, there being a tendency towards transition to uncrossed waves. Similarly, the transition of the shockfront geometry from crossed to uncrossed waves occurs for relatively weaker shocks as the aperture is decreased (cf. table 3). These effects are due to the fact that the convergence of the fronts from the sides and the nonlinear acceleration of the shock on the centre-line are competing effects. The waves cross if they come into the axis very steeply. On the other hand, if the convergence is slow and the stem shock in the focal region is strong, the shock accelerates out of the convergence, giving uncrossed waves. Thus, a rapid convergence suppresses nonlinear effects, whereas a slow convergence allows nonlinear effects to develop. Indeed, if distances are scaled with the focal length (as in figure 12) the nonlinear phenomena in the focusing of a shock wave depend only on the aperture of the convergence and the shock strength (including, of course, the possibility of shockstrength variation along the initial front).

3.1.4. Focus of an axisymmetric reflector. Experiments have also been conducted with an axisymmetric reflector (no. 4) which produces a point focus. In this case acoustic theory predicts that the focused wave should emerge as an expansion discontinuity. Furthermore, even the diffracted wavefronts focus on the axis as they cross it, producing a jump discontinuity (Cornet 1972). The sharp edge of the paraboloidal reflector is a circle and the diffracted front emanating from it is a growing torus; all rays emanating from the perimeter of the reflector with a fixed inclination to the axis converge at a point on the axis, and different points on the axis represent foci for rays with different inclinations. Nevertheless, if projected onto a plane containing the axis, the wavefronts look the same as those at a perfect line focus. 
Indeed, it is observed in our experiments that shockfronts near a point focus behave qualitatively in very much the same way as at a line focus, and show similar changes with shock strength. However, the critical shock strength for the transition in the geometry of the shockfronts is lower in this case, owing to the more rapid amplification in axial focusing (cf. table 3 ).

In order to demonstrate the difference between point and line foci, pressure histories on the axis beyond focus have been measured (figure 13, plate 5). In these experiments a dividing plane was not used, but the transducer was simply mounted on a sting support facing the reflected waves. Therefore, in figure 13 the pressure traces are influenced somewhat by diffraction over the body of the transducer. For weak shocks (top of figure) the diffracted fronts from the reflector corners arrive before the focused shock and the focused shock is inverted. Thus, the waveform has the appearance of a square pulse, consistent with the predictions of acoustics. The width of the square pulse is proportional to the size of the loop in the folded shockfront, which increases with distance from the focus and decreases with increasing shock strength. These traces may be compared with the corresponding ones of figure 7, for which linear theory predicts a logarithmic singularity. For the strongest shock (bottom of figure 13) the pulse width is much smaller, indicating that the probe is in the extended focal region; it is sensing the stem shock and the expansion wave behind it.

3.1.5. Approximate numerical simulation. The trajectories of the three-wave intersections have been computed based on a simplified geometrical model (Kulkarny 1975). The motion of the intersection of two fronts moving with different velocities is calculated numerically, assuming the difference in the velocities of the two fronts to be proportional to the shock strength. If the shock ahead of the intersecting wave has a Mach number $M$ (figure 14), then the intersecting wave is assumed to have a speed $(2 M-1) a_{0}$ independent of its amplitude and direction ( $a_{0}$ is the speed of sound in the fluid ahead of the shock). Further, if the angle $\alpha$ is known, the angle $\beta$ can be found as a function of $M$ and $\alpha$, which completely determines the motion of the intersection in this approximation. The intersection travels upwards along the incoming shockfront, exhibiting the primary nonlinear effect in the behaviour of the trajectories.

As the intersection travels along a converging shockfront, $\alpha$ and $\beta$ increase and tend to become large; this produces another effect. The shockfront, which is moving almost as rapidly as the intersecting wave, tends to 'run away' from the intersecting wave, creating a gap at the intersection, and the intersecting wave diffracts into this gap. This diffraction process curves the intersecting wavefront, continuously reorients the intersection and, most important, limits the maximum value of $\beta$. To account for this, in the numerical calculation $\beta$ is allowed to increase only to the value $\frac{1}{2} \pi$. As a consequence of this assumption, the speed of the intersection along the shock reaches a minimum value and then depends on $M$ alone.

To model the three-wave intersections for a reflector with a perfect line focus, the calculation of the intersections starts at the reflector corners and proceeds towards the focus. For simplicity, the shock is assumed to be circular and its initial strength to be constant along the front. Its strength at subsequent times is given 


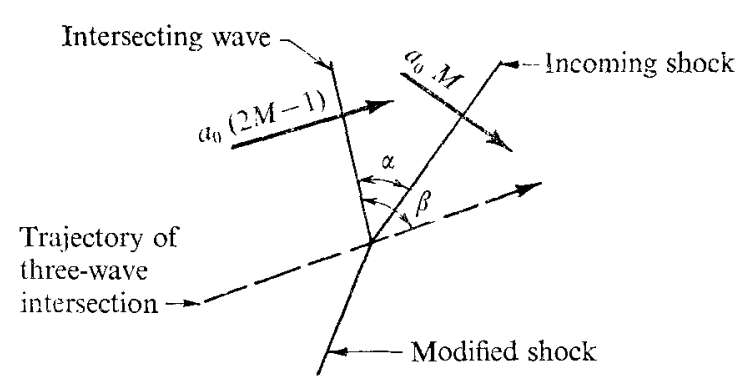

Figure 14. Model of a three-wave intersection.

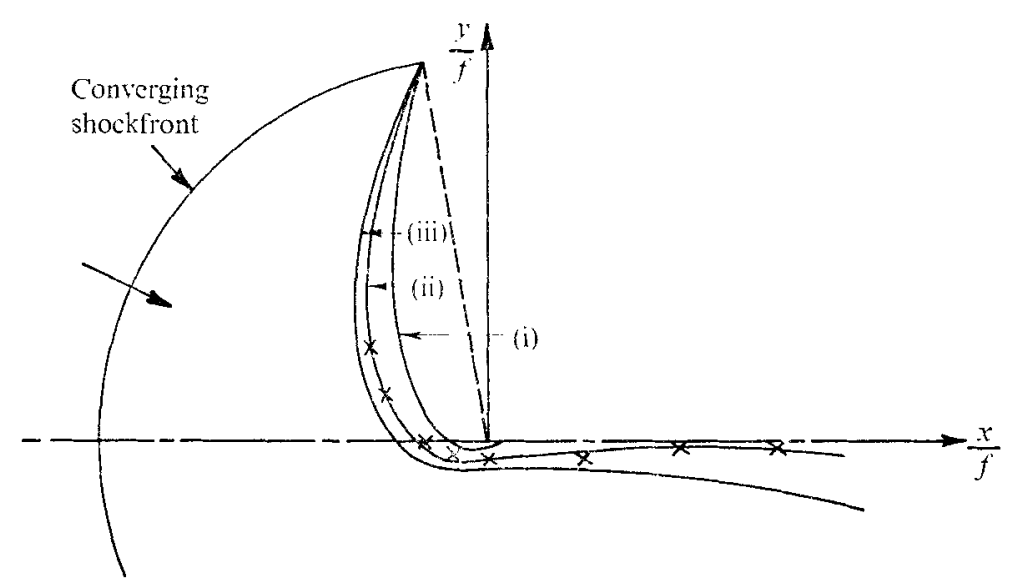

Figure 15. Trajectories of three-wave intersections produced by approximate numerical simulation; perfect focus. Reflector no. $1 . M$ : (i), 1.1; (ii), 1.2; (iii), 1.3. $\times$, Experiment, $M_{8}=1 \cdot 2$.

by geometrical acoustics. This determines the trajectories to the point where they meet on the axis. At this point the whole shockfront has been affected by the diffracted fronts so strictly speaking its shape and strength are not known. In order to complete the calculation, the following argument is made, to fix the shock shape. The diffracted compression fronts from the reflector corners are assumed to travel with speed $a_{0}$. This results in a misfit between the diffracted front and the converging shock. The misfit is eliminated by assuming that the two fronts are connected by a straight shockfront tangential to both of them. It is assumed that the strength of the composite shockfront varies smoothly from maximum on the centre-line (constant and equal to the value calculated jjust at the crossing of the trajectories) to zero at the diffracted front.

Figure 15 shows the results of some calculations for reflector no. 1 and a comparison with the trajectory shape obtained from the shadowgraphs for $M_{s}=1 \cdot 2$ The computed Mach numbers for transition from crossed to uncrossed fronts for other reflectors are tabulated in table 3 . The agreement is excellent, indicating that the basic cause of the behaviour of the three-wave intersections is indeed nonlinear gasdynamic effects. 


\subsection{Arête}

3.2.1. Shadowgraphs. Figure 16 (plate 6) shows four successive stages of a focusing shockfront near an arête. This sequence represents the behaviour of a weak shock at an arête, as signalled by the crossed wavefront configuration in the last picture. None the less, occurrence of strong shocks near the focus is evident from the heated fluid (dark, peninsular region in the centre).

Figure $16(a)$ shows the reflected shock (dark curve, concave to the right) approaching the cusp of the caustic. Near the most concave portion of the shockfront, some evidence of diffraction can be seen. This is quite expected, since the most concave portion is also the most amplified portion, being nearest to a focus. In figure $16(b)$ the shockfront has just passed the cusp predicted by geometrical acoustics. In this frame the diffraction is seen more clearly. It is of the form of a compression wave followed by an expansion wave, and is the same wave field behind the incident shockfront as was seen in the perfect focus. In figure $16(c)$ the compressive portions nearest the axis have steepened to form diffraction shocks. These shocks intersect with the incident shock to form two three-shock intersections with a common stem shock. In the figure strong refraction of light has masked the stem shock in the centre, but its span is indicated by the separation between the slipstreams of the two three-shock intersections (two pairs of dark and bright lines, trailing in the fluid). Further development of the flow field is shown in figure $16(d)$. The reflected shockfront has crossed ahead of the focused shock, thus forming a triangular loop in the shockfront. The diffraction shocks now meet the two off-axis apices of this triangle, making three-shock intersections at those points. The slipstreams of these intersections have crossed in the fluid behind the shockfronts.

Thus, as before, nonlinear diffraction processes behind the shockfront lead to the formation of two shock-shocks. Then the shock-shocks cross, after which the shockfronts are crossed and folded. The only major difference between this behaviour and that of the perfect focus is that in the present case the diffracted expansions behind the focusing shock are not distinct (since there are no sharp corners to generate them), with the result that the shock-shocks start far apart. Consequently, there is no distinct point marking the beginning of the focal spot.

Figure 17 (plate 7) shows shadowgraph sequences for four different incident shock strengths. It may be seen that, with increasing shock strength, the focal region becomes larger and the triangular loop smaller. Finally, as shown in the last sequence, the shock-shocks do not cross, but simply spread apart, leaving a focal region of indefinite extent. The final shockfront is not crossed, and there is no triangular loop. This behaviour is completely analogous to the behaviour near perfect foci.

It is interesting to note that the slipstreams of the three-shock intersections for $M_{s}=1.5$ become turbulent a short distance behind the intersections. The last picture of the sequence shows the starting point of the slipstreams long after departure of the wavefronts. Being shear layers, they become unstable and roll up into two line vortices. The layers beyond the vortices are turbulent and merge together to form a jet. 


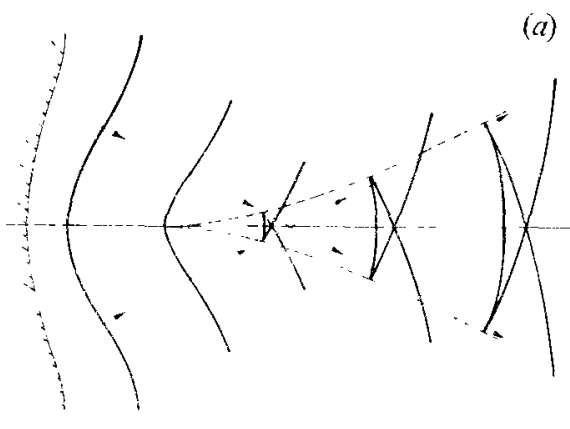

(b)

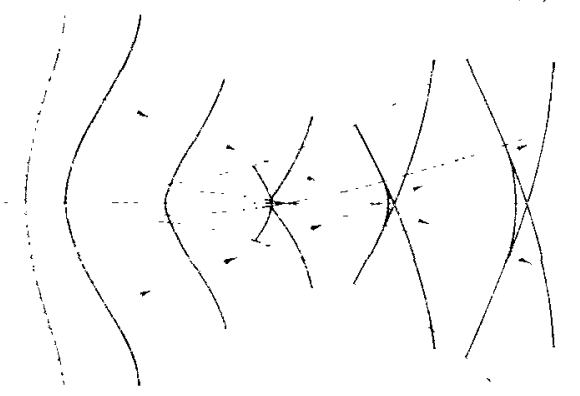

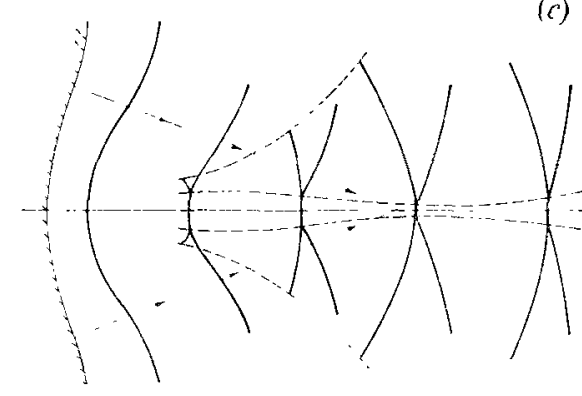

(d)

FIGURE 18. Schematic representation of the effect of shock strength on focusing. Arête of: (a) sound pulses; (b) weak shocks; (c) moderately strong shocks; (d) strong shocks. ----, shock-shocks and the loci of the free extremities of the diffraction shocks.

The behaviour of (a) sound pulses, (b) weak shocks, $(c)$ moderately strong shocks, and $(d)$ strong shocks near an arête are summarized schematically in figure 18. The shock-shocks in each case are shown by a pair of dashed lines close to the axis of symmetry. A second pair of dashed lines marks the loci of the free extremities of the diffraction shocks. In the acoustic limit they all coincide with the two branches of the caustic. With finite amplitude, the locus of the shock extremity shifts towards the centre of curvature of the caustic, while the shockshocks shift away from it. Consequently, the shock-shocks cross on the axis and form the narrow peninsular focal region. The arrows in the figure indicate the motion of the inflexion points of the shockfront. The angle enclosed by these lines represents the effective angle of convergence of the shockfront.

It is important to note that the region between the shock extremity locus and the corresponding shock-shock is a focal region of the caustic inside it. That is, we speak of two focal regions: the first in the neighbourhood of the arête, which is similar to a perfect focus, and the second at the caustics, which for large time becomes quasi-steady.

3.2.2. Pressure histories. Some typical pressure traces observed in the different regions of the flow field are presented in figure 19 (plate 8). The bottom row of traces shows that along the axis the shock jump becomes greatly magnified, but is imrnediately followed by a sharp expansion which by nonlinearity overtakes 

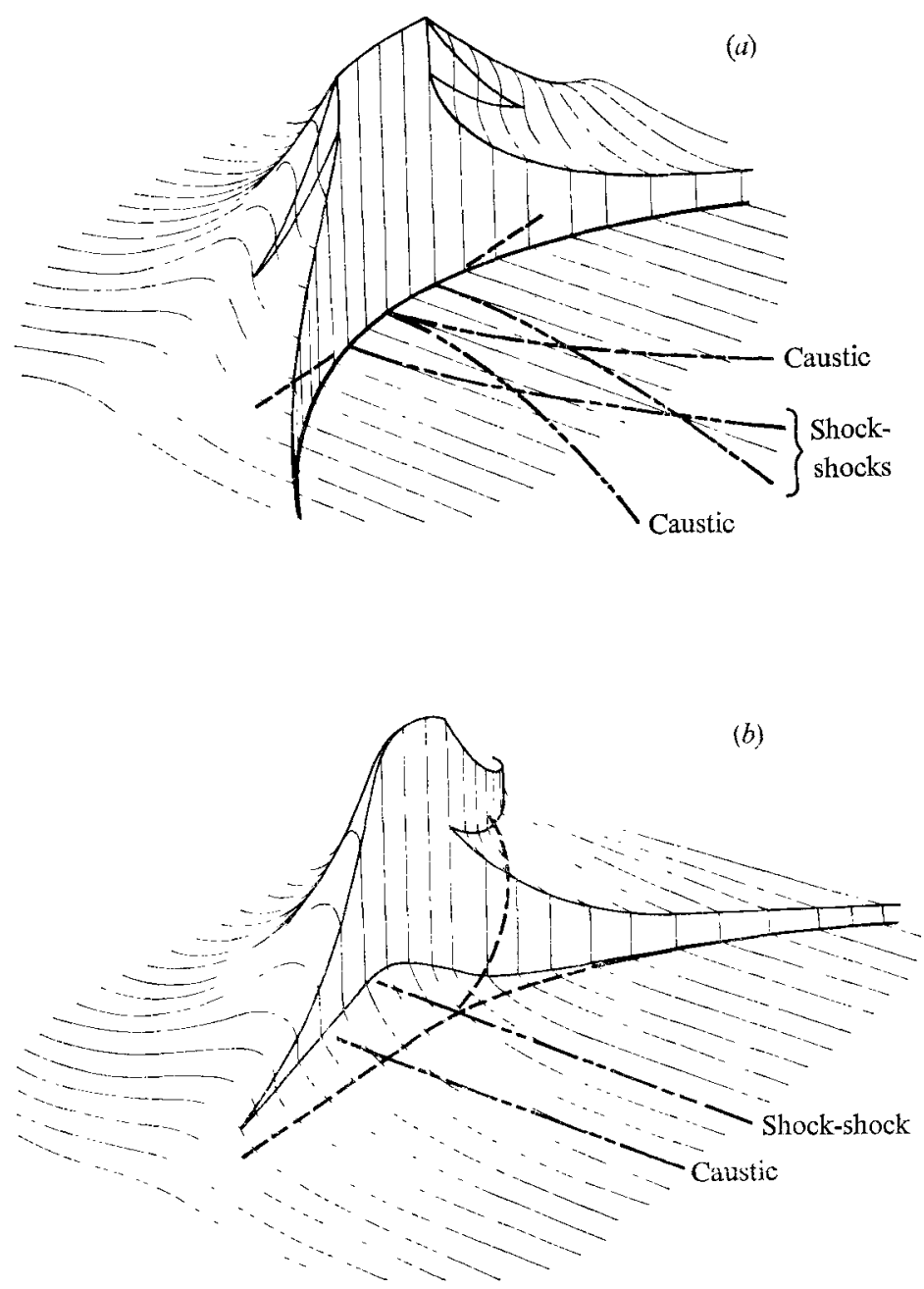

FraURt 20. Qualitative isometric views of the pressure field near the focal region of (a) arête, (b) caustic. The wavefronts are shown with heavy lines on the ground travelling towards and to the right of the observer, and the pressure is shown vertically upwards.

the shock and controls its amplitude in the focal region. The top row shows the development of the compression-expansion field behind the initial front. In the focal region of the caustic (top row, last picture) the diffracted expansion that follows the diffraction shock also overtakes this shock and controls its amplitude. For strong shocks, the focal region of the cusp extends to infinity, and the focus of the caustic never develops.

Figure 20 shows by isometric view the qualitative nature of the pressure field in the two important focal regions. (Figure $20(b)$ is a view of the field near the caustic at the bottom right of figure 19.) In both regions the shock amplitude is controlled by an overtaking expansion. Also, both sketches show the compression-expansion that gradually becomes steeper near a focus and forms a 

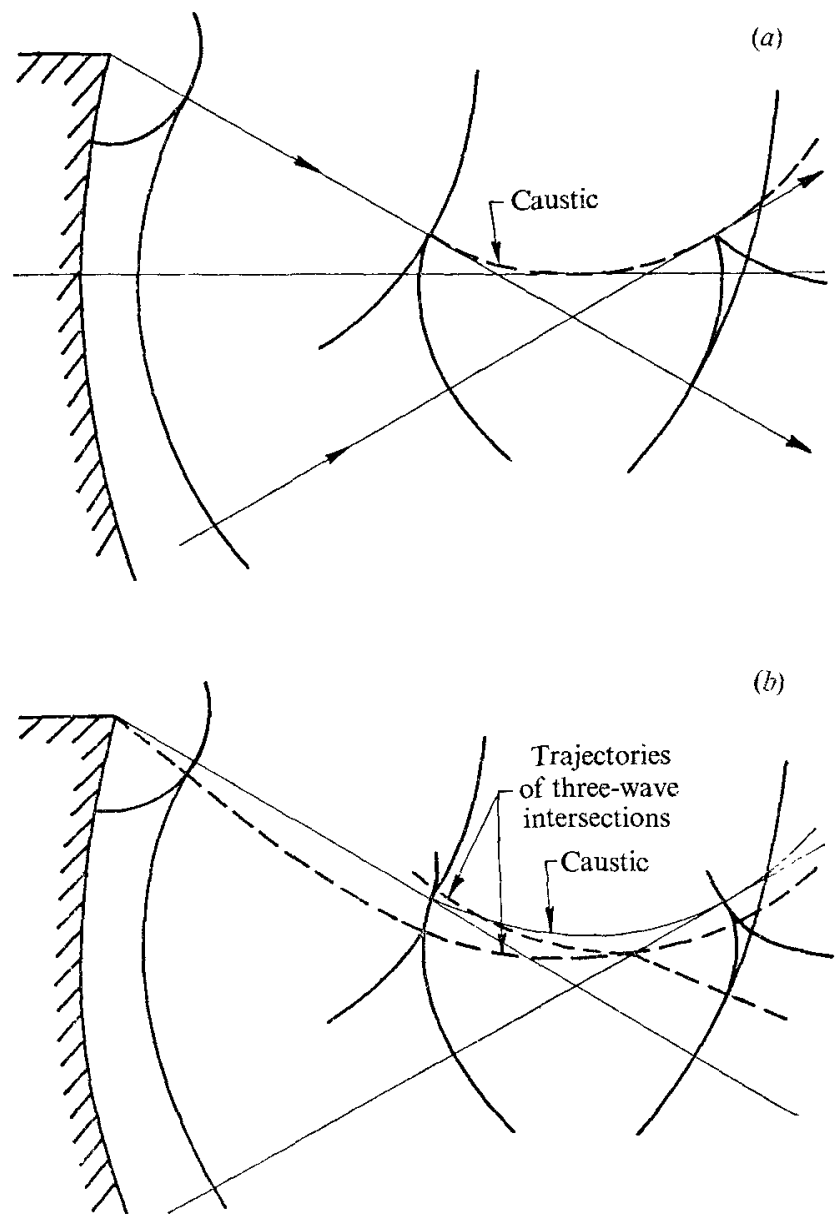

FIGURE 22. Schematic representation of the effect of shock strength on focusing. Caustic of (a) a sound pulse, (b) a weak shock.

diffraction shock with a free extremity. In figure $20(a)$ this shock is the reflected shock of a three-shock intersection, while in figure $20(b)$ it is the stem shock.

\subsection{Caustic}

In this section, experiments with reflector no. 5, which produces a caustic with a constant radius of curvature, are reported. The discontinuity in shockfront radius of curvature necessary to generate the starting point of the caustic arises from the sharp corner of the reflector (cf. figure $22(a)$ ). Figure 21 (plate 9) shows shadowgraphs of shock waves at successive points along the caustic for two different shock strengths. The caustic is an arc of a circle, concave up, in the upper part of the pictures. In the sequence for the weak shock, the first picture shows the converging shockfront (wave at lower right) just starting to cross the diffracted compression from the reflector corner (upper left). The crossing is an essential feature of the starting point of a caustic. The wave behind the shock- 
front (on the left) is the diffracted expansion from the corner. In the second picture the front is crossed and folded, and the reflected, or focused, shock completing the triangular loop may be seen. The upper apex of the triangular loop travels along the caustic. When the cross and the other fold of the loop move away, the processes at the caustic become steady.

In the sequence for the stronger shock, the details of both the initial crossing and the focal region of the caustic can be seen more clearly. In the first picture, a diffraction front is seen on the upper (concave) side of the caustic; along its upper portion near the free extremity it is a smooth front, but lower down it has steepened into a diffraction shock. The diffraction shock forms a three-shock intersection with the diffracted compression from the corner, as indicated by the upper slipstream. The diffracted expansion from the corner is also preceded by a diffraction shock (formed by the same process), which results in another threeshock intersection, and the lower slipstream. It is interesting to note that at this stage the waves in the upper part of the picture are similar to those that occur at an arête, whereas in the lower part they are comparable to the waves at a perfect focus. The remaining pictures show the subsequent crossing of the shockfront, and the growth of the focal region of the caustic. This behaviour is indicated schematically in figure 22 for a sound pulse and for a weak shock.

\section{Conclusions}

The experimental results presented in this paper demonstrate that the behaviour of a shock discontinuity at a variety of different kinds of focus is determined by the same gasdynamic processes in all cases. Though focusing weak waves emerge with crossed and looped fronts while strong waves do not, nonlinear gasdynamic effects always dominate near the focus. Their function is to determine the wavefront geometry at and beyond focus, and to limit the maximum amplitude at focus. The resulting wave interactions near a focus are much"more complex than was previously thought to be the case.

The wavefront geometry is determined in"all cases by two predominant nonlinear effects. First, the diffracted field behind the non-uniform converging front steepens to form a diffraction shock, and, second, the diffraction shock participates at different stages of the focal process either as the reflected shock or the Mach stem of a Mach reflexion. In the former case (i.e. during the early, transient stages of the focal process), the development of the Mach reflexion, depending, as it does, on competition between the convergence of waves from the sides and acceleration of the shock on the centre-line, determines whether the three-shock intersections cross or remain uncrossed. If they cross, the wavefronts also cross; the wavefront crossing is similar to acoustic regular reflexion, and in this case the diffraction shock becomes a Mach stem. If they do not cross, the diffraction shock remains the reflected shock of the Mach reflexion.

In all cases the amplitude near focus is limited by the overtaking of a Mach stem by a diffracted expansion wave. A problem not treated in the present experiments is that of the variation of shock strength along the Mach stem, particularly near the three-shock intersection. The behaviour near the three-shock inter- 
section is especially important near the steady focus of a caustic (e.g. figure $20(b)$ ) because it falls close to the singularity of geometrical acoustics. In this case maximum pressures will be found near the three-shock intersection, so this is the region of major interest. However, the flow at a three-shock intersection remains one of the unsolved problems of fluid mechanics; we can only say that it evidently plays an important role near the focus of a shock wave.

The authors are grateful for the support of this work by the Air Force Office of Scientific Research under grant 71-2092.

\section{REFERENCES}

Beasley, W. D., Brooks, J. D. \& Barger, R. L. 1969 N.A.S.A. Tech. Note, D-5306.

Bleakney, W. \& TAUB, A. H. 1949 Rev. Mod. Phys. 21, 584.

Brtscoe, M. G. \& Kovitz, A. A. 1968 J. Fluid Mech. 31, 529.

ConNet, E. P. 1972 Appl. Res. Labs. University of Texas, Rep. no. 72.40.

Friedlander, F. G. 1958 Sound Pulses. Cambridge University Press.

Friedman, M. B. 1968 N.A.S.A. Rep. SP-180, p. 115.

Friedman, M. P. \& Chov, D. C. 1965 N.A.S.A. Rep. CR-358.

Friedman, M. P., Kane, E. J. \& Sigatida, A. 1963 A.I.A.A. J. $1,1327$.

GILL, P. M. 1973 Nonlinear acoustics behaviour at a caustic. Ph.D. thesis, Cornell University.

Gutraud, J. P. 1965 J. de Méc. 4, 215.

HaYes, W. D. 1968 N.A.S.A. Rep. SP-180, p. 165.

Keller, J. B. 1954 J. Appl. Phys. 25, 938.

Keller, J. B. \& Blank, A. 1951 Comm. Pure Appl. Math. 4, 75.

KULKARNY, V.A. 1975 An experimental investigation on focussing of weak shock waves in air. Ph.D. thesis, California Institute of Technology.

Liepmann, H. W., Roshro, A., Coles, D. E. \& Sturtevant, B. 1962 Rev. Sci. Instr. 33, 625.

Natmann, A. \& Hermanns, E. 1974 Noise Mechanisms, AGARD Conf. Proc. 131, paper 23.

Obermeier, F. 1974 Noise Mechanisms, AGARD Conf. Proc. 131, paper 17.

SaNat, M. \& Toong, T. Y. 1974 2nd Interagency Symp. on Univ. Res. in Transport Noise, North Carolina State University, Raleigh, p. 416.

Seebass, R., Murman, E. M. \& Krupp, J. A. 1970 N.A.S.A. Rep. SP.255.

Thery, C., Peter, A. \& Pfister, M. 1970 Institut Franco-Allemand de Recherches de Saint Louis, N. T. 9/70.

Wanner, J. C., Vallee, J., Vivier, C. \& Thery, C. $1972 J$. Acoust. Soc. Am. 52, 13.

Whiteam, G. B. 1957 J. Fluid Mech. 2, 145.

WhITHAM, G. B. 1959 J. Fluid Mech. 5. 369. 